nicks. Members of this ubiquitous family of structurespecific endonucleases function as dimers and require divalent cations for cleavage. We have solved the crystal structures of cruciform cutting enzyme 1 (CCE1) from Candida glabrata and Holliday junction cutting enzyme (Hjc) from the hyperthermophile archaeon Archaeoglobus fulgidus [1] at 3 and $1.7 \AA$ resolution, respectively. They represent two structurally distinct resolvase families with the same biological function, but exhibiting clearly different substrate specificities. Co-crystallization of these proteins with selected DNA substrates is under way.

[1] Biertumpfel, C., Basquin, J., Birkenbihl, R.P., Suck, D., Sauter, C. Acta Crystallography, 2005, F61, 684.

\section{MS03 P04}

The Protease State of the Heat-Shock Protein DegP

from Escherichia coli Tobias Krojer, Justyna Sawa, Fuhrmann Jakob, Gazda Linn, Kirk Rebecca, Kurt Juliane, Nussbaumer Barbara, Schmidt Bastian and Tim Clausen, Research Institute of Molecular Pathology (IMP), Dr. Bohrgasse 7, A-1030, Vienna, Austria.

E-mail: krojer@imp.univie.ac.at

\section{Keywords: DegP, protease, chaperone}

DegP (a.k.a. HtrA, protease Do) is a heat-shock protein which is localized in the periplasm of Escherichia coli. It is an essential, ATP-independent protein quality control factor and represents the only known protein that can alternate between the two antagonistic activities of a protease and a chaperone in a temperature-dependent manner [1]. The mature protein consist of a chymotrypsinlike serine protease domain and two consecutive, Cterminal PDZ domains. DegP is present as a hexamer in solution and belongs to the diverse family of cage-forming proteases. The active sites are shielded from the environment in an inner cavity, to prevent the unwanted degradation of functional proteins. The crystal structure of the chaperone conformation of DegP revealed that substrate degradation at low temperatures is additionally abolished by a distorted and blocked active site [2].

Here we present the crystal structure of DegP in complex with the small molecule inhibitor diisopropyl fluorophosphates (DFP). DFP did not lock the active sites in the canonical conformation of chymotrypsin-like serine proteases. The inhibitor is rather bound in an atypical conformation, which was never observed before in serine proteases, again underlining the remarkable flexibility of the active site. Furthermore, time-dependent analysis of degradation products by HPLC chromatography revealed that $\mathrm{DegP}$ acts as a processive protease, similar to other cage-forming proteases like the proteasome or ClpP. Analysis of degradation products of DegP by mass spectrometry confirmed that $\mathrm{DegP}$ has a clear preference for aliphatic residues in the P1 site. Furthermore, the product spectrum shows a normal distribution with a mean peptide length of 13 to 15 residues.

In summary, our results suggest that the proteolytic active sites of DegP exhibit an unusual degree of flexibility, which may be necessary to allow the efficient degradation of a wide variety of different, misfolded proteins before those become harmful for the cell. The observed product length distribution and the processive degradation of substrate proteins refer to yet unidentified trans-acting determinants which are essential for substrate binding and degradation.
[1] Spiess C., Beil A., Ehrmann M., Cell, 1999, 97(3), 339.

[2] Krojer T., Garrido-Franco M., Huber R., Ehrmann M., Clausen T., Nature, 2002, 416, 455.

\section{MS03 P05 \\ Identity of Ions in the Second Site of Subtilisins \\ Jan Dohnalek ${ }^{\mathrm{a}, \mathrm{b}}$, Allan Svendsen ${ }^{\mathrm{c}}$, Keith S. Wilson ${ }^{\mathrm{d}}$, ${ }^{a}$ Institute of Macromolecular Chemistry of the Academy of Sciences of the Czech Republic, Heyrovskeho nam. 2, 162 06 Praha 6, Czech Republic. ${ }^{\mathrm{b}}$ Institute of Physics of the Academy of Sciences of the Czech Republic, Cukrovarnicka 10, 16253 Praha 6, Czech Republic. ${ }^{\mathrm{c}}$ Protein Design, Novozymes A/S, Brudelysvej 26,DK-2880 Bagsveerd, Denmark. ${ }^{\mathrm{d} S t r u c t u r a l}$ Biology Laboratory, University of York, Heslington, York, YO10 5YW, United Kingdom. E-mail: dohnalek@imc.cas.cz}

\section{Keywords: subtilisin, serine protease, calcium binding}

Serine proteases classified under the EC number 3.4.21.62 - subtilisins - have been extensively studied by x-ray crystallography with focus on the relationship between metal binding and stability. These $\alpha / \beta$ hydrolases with broad specificity typically bind one or more calcium ions and in some cases other, mostly monovalent, ions [1]. Subtilisins BPN'/NOVO, Carlsberg or Savinase were studied systematically as for their stability related to metal binding since 1969. Two typical sites - the strong calcium binding site (Ca-I, formerly named site A) and the weak calcium binding site (Ca-II, formerly named site B) were identified. Stability of subtilisin BPN' depends on the presence of site Ca-I whereas the stabilization effect of site Ca-II depends on the concentration of ions in the solvent [2]. Site Ca-II is accompanied by a closely related site Na-II only $2.6 \AA$ away, with high affinity for sodium. Our detailed analysis of the contents of sites Ca-II and NaII as present in the published structures leads to the conclusion that in some cases their contents were misinterpreted. We have reclassified the typical metal binding sites of subtilisins and subtilisin-like proteases and proposed the most likely identities of the ions and atoms bound in sites Ca-II and Na-II in the publicly available structures.

This work was supported by Novozymes, Denmark and by the Ministry of Education, Youth and Sport of the Czech Republic (project no. 1K05008).

[1] Siezen R.J., Leunissen J.A.M, Prot. Sci., 1997, 6, 501.

[2] Alexander P.A., Ruan B., Bryan P.N., Biochemistry 2001, 40, 10634.

\section{MS03 P06}

The Enolase in the Methionine Salvage Pathway: Crystal Structure and Function. A.Fedorov, H.Imker, E.Fedorov, J.Gerlt, S. Almo. Department of Biochemistry, AECOM, New York,USA. E-mail:fedorov@aecom.yu.edu

\section{Keywords:Enolase, RuBisCO, Crystal structure}

D-Ribulose1,5-biphosphate carboxylase/oxygenase (RuBisCO), the most abundant enzyme, is the paradigm member of the recently recognized mechanistically diverse RuBisCO superfamily. The heterofunctional homologue of $\mathrm{RuBisCO}$ found in Geobacillus kaustophilus catalyze the enolization of 2,3-diketo-5-methylthiopentane 1-phosphate in the methionine salvage pathway. Because the RuBisCO and the enolase - catalyzed reactions differ we sought to 